\title{
Psychological effects of generalized nutritional deprivation in the elderly
}

\author{
Tommy Cederholm \\ Department of Geriatric Medicine, Huddinge University Hospital, Stockholm, Sweden
}

\section{Abstract}

The Minnesota starvation study from the mid 1940s provides convincing evidence that imposed nutritional deprivation and weight loss induce depression, irritability and asociability. Similar findings are recently reported in elderly institutionalized and home-living malnourished populations, although confounding effects of concurring disease cannot be excluded. Several key nutrients influence mood, e.g. carbohydrates, tryptophane, vitamin $\mathrm{B}_{12}$, folate, thiamine and iron, and it is reasonable to believe that the detrimental psychological effects of generalized malnutrition are a combined effect of such key nutrient deficiencies. The few nutrition intervention studies in malnourished elderly, using a balanced nutrient supply, report conflicting data on psychological effects.

Keywords: depression; elderly; protein-energy malnutrition; quality of life

\section{Introduction}

Protein-energy malnutrition (PEM), i.e. weight loss and underweight, commonly occurs in chronically ill and elderly subjects. Lots of evidence links PEM to increased somatic morbidity, e.g. susceptibility to infections (1) and increased mortality. The psychological effects of PEM have not been explored to the same extent (2), although the effects of deficiencies of single key nutrients on mood are fairly well characterized (3). Various established instruments for the assessment of well-being and quality of life (QoL), e.g. the Short-Form 36 health survey questionnaire (SF-36), Nottingham Health Profile (NHP), Sickness Impact Profile and Euroqol (4), have been used in studies on nutritional deprivation. Perceived QoL is determined by an array of factors. For example, pain, dyspnoea and bereavements are factors related to disease and ageing, i.e. the usual underlying causes for PEM. Although the causal relationships underlying impaired QoL are complex it is important to elucidate the mental status and perception of QoL during PEM to understand the complex consequences of PEM.

\section{The Minnesota starvation study}

After nearly 60 years the Minnesota Starvation Study still provides some of the strongest evidence for the importance of nutrition in mental function $(2,5)$. In the late phase of the Second World War 34 young objectivees voluntarily reduced their food intake to $1500 \mathrm{kcal} \mathrm{day}^{-1}$ for 6 months and lost an average of $25 \%$ of their body weight. As well as expected findings such as muscle fatigue and tiredness, psychological and emotional problems appeared in particular. Extravert and lively men became apathic, irritable and socially withdrawn. The mean Depression score ( $0-100$ points) used in the study rose from the normal 53 points to 72 points (Fig. 1). When the nutritional status slowly became normalized after the semi-starvation period had ended the psychological state improved, but was not restored until after 9 months.

\section{Psychological effects of malnutrition in institutionalized elderly: chicken or egg?}

The Minnesota starvation study gives us an understanding of the effects of food deprivation per se on psychological-mental performance. Although the Minnesota study investigated young men, it is reasonable to believe that women and the elderly would react in a similar fashion. Generalized nutritional deprivation, especially in affluent societies, seldom occurs without an underlying cause, e.g. disease. Thus, in depleted, chronically ill and 


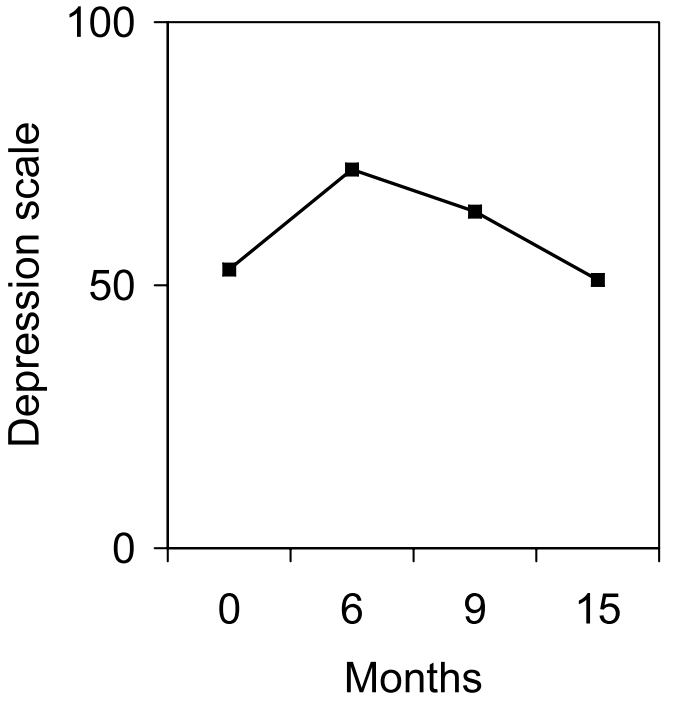

Fig. 1. Effect on self-estimated depression from semi-starvation for 6 months followed by 9 months of refeeding in 34 volunteers. Higher scores indicate worse depression (50 points is considered normal).

depressed elderly subjects the causal relationships become difficult to determine. Studies that have addressed psychological-nutritional relations in the elderly are mainly performed in hospitalized or institutionalized subjects.

Morley and Kraenzle found in a population of 200 nursing-home residents (mean age 80 years) that 30 subjects had lost $>2 \mathrm{~kg}$ of weight during the past 6 months. Sixty per cent of these were assessed as depressed and the conclusion was that depression was a common cause of weight loss (6). Interestingly, it was reported that most of these residents were conventionally treated for depression but appeared resistant to treatment. One may speculate whether the depression was secondary to malnutrition rather than the reverse. In a study of 205 hospitalized patients (mean age 75 years) with non-malignant disorders, the patients were asked to assess their mood according to a seven-graded scale. Malnourished women assessed their mood as depressed compared with well-nourished women (7). Linear regression analysis disclosed chronic disease and PEM as independent predictors for a depressive mood. In another Swedish study of 199 surgical patients (mean age 59 years, $40 \%$ gastrointestinal malignancy) the nutritional status was related to a QoL instrument that contained 18 various domains (8). Several of the domains, e.g. mood, anxiety and endurance, were related to impaired nutritional status. Weight loss and low serum albumin appeared as the strongest predictors of reduced QoL. Fortytwo women $>70$ years of age acutely admitted to an orthopaedic service owing to recent hip fracture were asked to assess their mood and to fill in the NHP, a six-item questionnaire (9). Every third woman had a body mass index (BMI) $<20 \mathrm{~kg} / \mathrm{m}^{2}$. The thin women assessed themselves as sad more often than patients with $\mathrm{BMI}>20 \mathrm{~kg} / \mathrm{m}^{2}$ did. Furthermore, the total NHP score indicated significantly reduced QoL in the underweight patients. Lack of energy, one of the six domains of the NHP, was the most prominent finding (Fig. 2).

\section{Nutrition and mood in home-living elderly}

In a recent survey of 353 home-living elderly recipients of community-based home help the nutritional status was classified as malnourished, at risk for malnourishment and well-nourished using the Mini Nutritional Assessment (10). About $20 \%$ of the well-nourished subjects reported a depressive mood, in line with results from population-based investigations of the elderly in general in Sweden and elsewhere (11). However, half of the malnourished subjects reported that they were very depressed or often depressed. Similar findings have been reported in a group of 155 American homeliving elderly subjects who received wheels-on-meals service, where the degree of nutritional risk, depres-

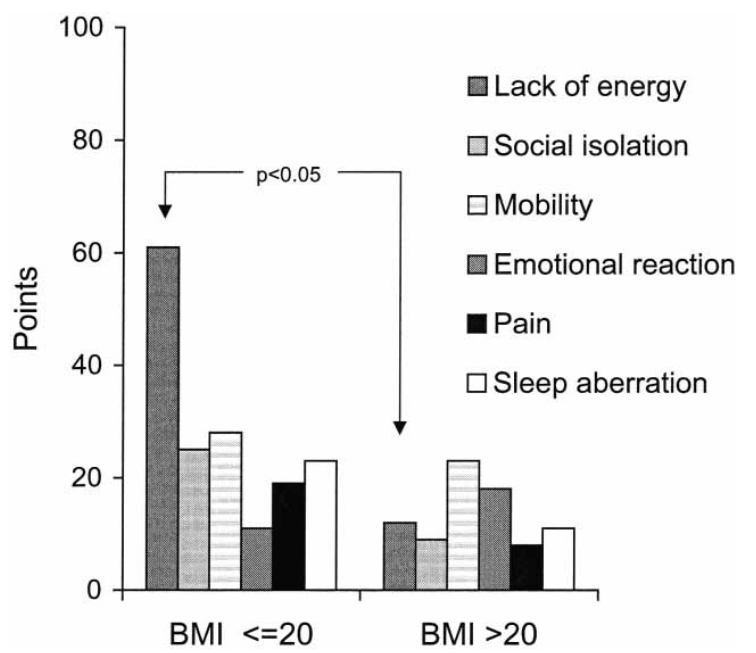

Fig. 2. Self-estimated quality of life according to the Nottingham Health Profile ( $0-100$ points) in elderly women with recent femoral neck fracture and divided by body mass index (BMI) more or less than $20 \mathrm{~kg} / \mathrm{m}^{2}$. A high score corresponds to a worse outcome. 
sion and perceived QoL correlated significantly (12).

\section{Macronutrient or micronutrient deficiency?}

Several nutrients are postulated to influence brain signal transduction pathways involved in emotional behaviour (3). Among macronutrients carbohydrate intake has been suggested to improve mood by influencing the bioavailability of tryptophan, the amino acid precursor of serotonin. Carbohydrateinduced postprandial insulin secretion favours the peripheral uptake of amino acids other than tryptophan, leaving a relatively high amount of tryptophan to be utilized by the brain. Low serum levels of $n-3$ fatty acids (e.g. fish oil) have been reported in patients with depression (13) and treatment with $\mathrm{n}-3$ fatty acids has proven effective in depressed patients resistant to standard therapy (14).

The relationships between micronutrient deficiencies and mood have been investigated more extensively. Hyperhomocysteinaemia related to folate or vitamin $\mathrm{B}_{12}$ deficiency in the elderly is a common occurrence and is followed by depressed mood that improves with substitution therapy (3). Similar findings with poor thiamine status (vitamin $\mathrm{B}_{1}$ ) have been reported, and are also true for iron deficiency. In elderly people with a poor nutritional status it is reasonable to believe that the nutritional contribution to a depressed mood comes from the combined action of several parallel nutrient deficiencies.

\section{Effects on personality by nutritional therapy}

A few studies have addressed the question of the psychological effects of treating malnourished subjects. Fifty-four geriatric patients (mean age 85 years) at risk for malnutrition were treated after discharge from hospital with dietary counselling, e.g. recommendations to increase fat intake, and a balanced liquid formula in the form of Semper Komplett $^{\circledR}$ and a multivitamin preparation, Friggs ${ }^{\circledR}$ (15). The SF-36 questionnaire showed that the study group classified their QoL as lower than did Swedish elderly people in general. Preliminary data indicate that, despite improved functions in activities of daily living (ADL), the nutritional intervention did not improve the outcome in terms of QoL. Elevated serum homocysteine levels were not normalized during the study period. In another ongoing study, 59 lean women (mean age 83 years) treated for recent hip fracture were randomized to 6 months of daily treatment with a liquid formula $\left(\right.$ Fortimel $^{\circledR}$ ) alone or in combination with the anabolic hormone nandrolone (Deca-Durabol ${ }^{\circledR}$ ), administered every third week (16). Preliminary results show that patients who received the formula did not display an equally pronounced decline in perceived QoL, as assessed by Euroqol, as the control patients. The best effect on QoL was achieved in the patients who were given the combined treatment.

Megestrol acetate, an antioestrogen preparation originally used for the prevention of mammary cancer relapses, has become increasingly popular as an appetite-stimulating drug. The appetite-stimulating effect of the drug is attributed to antiinflammatory effects, e.g. tumour necrosis factor generation is inhibited. Megace ${ }^{\circledR}$ has mainly been used as an orexigenic drug in patients with cancer or human immunodeficiency virus (HIV) cachexia. However, in one study 50 depleted American nursing-home residents (mean age 76 years) were randomized to megestrol acetate treatment for 12 weeks. Together with improved appetite and small effects on weight, the patients reported a better sense of well-being (17).

\section{Conclusion}

Owing to the complex interactions between malnutrition, disease, frailty and emotional status, which often co-occur, it is difficult to draw firm conclusions about their causal relationships. The reviewed evidence strongly indicates that PEM in older adults negatively affects mood and perceived QoL, probably as an effect of parallel deficiencies of several key nutrients. Keeping good nutritional routines to maintain the nutritional status in the frail elderly and chronically ill is a challenge for the health system as well as for the community care system which, if met adequately, may have a substantial impact on well-being in the late phases of life. Further studies are need to determine how the nutritional support should be composed in terms of macronutrient and micronutrient content to have optimal effects on maintaining or restoring psychological functions. Health and nutritional status are in many cases not the most important determinants of QoL, but QoL is an aspect of the treatment outcome that should be taken into account in nutritional treatment studies. 


\section{Acknowledgements}

This review was compiled with support from the Swedish Research Council.

\section{References}

1. Pennington CR. Disease-associated malnutrition in the year 2000. Postgrad Med J 1998; 74: 65-71.

2. Brozek J. Effects of generalized malnutrition on personality. Nutrition 1990; 6: 389-95.

3. Benton D, Donohoe R. The effects of nutrients on mood. Publ Health Nutr 1999; 2: 403-9.

4. Andresen EM, Rothenberg BM, Panzer R, Katz P, McDermott MP. Selecting a generic measure of healthrelated quality of life for use among older adults. A comparison of candidate instruments. Eval Health Prof 1998; 21: 244-64.

5. Keys A, Brozek J, Henschel A, Mickelsen O, Taylor L. The biology of human starvation. Minneapolis, MN: University of Minnesota Press; 1950.

6. Morley J, Kraenzle D. Causes of weight loss in a community nursing home. J Am Ger Soc 1994; 42: 583-5.

7. Cederholm T, Jägren C, Hellström K. Nutritional status and performance capacity in internal medical patients. Clin Nutr 1993; 12: 8-14.

8. Larsson J, Åkerlind I, Permerth J, Hörnqvist J-O. The relation between nutritional state and quality of life in surgical patients. Eur J Surg 1994; 160: 329-34.

9. Ponzer S, Tidermark J, Brismar K, Söderqvist A, Cederholm T. Nutritional status, insulin-like growth factor-1 and quality of life in elderly women with hip fracture. Clin Nutr 1999; 18: 241-6.

10. Saletti A, Yifter-Lindgren E, Wissing U, Österberg K, Johansson L, Cederholm T. Nutritionl problems in elderly with home help. The National Board of Health and Welfare. Äldreuppdraget 99:5. (In Swedish.)

11. Gottfries CG. Late life depression. Eur Arch Psychiatry Clin Neurosci 2001; 251(Suppl 2): I57-61.

12. Vailas LI, Nitzke SA, Becker M, Gast J. Risk indicators for malnutrition are associated inversely with quality of life for participants in meal programs for older adults. $\mathbf{J}$ Am Diet Assoc 1998; 98: 548-53.

13. Mamalakis G, Tornaritis M, Kafatos A. Depression and adipose essential polyunsaturated fatty acids. Prostaglandins Leukot Essent Fatty Acids 2002; 67: 311-8.

14. Peet M, Horrobin DF. A dose-ranging study of the effects of ethyl-eicosapentaenoate in patients with ongoing depression despite apparently adequate treatment with standard drugs. Arch Gen Psychiatry 2002; 59: 913-9.

15. Persson M, Hytter $\AA$, Cederholm T. Effects of dietary counselling and liquid supplementation on weight, ADL function and serum lipids in malnourished geriatric outpatients (Abstract). Clin Nutr 2000; 19(Suppl 1): 2009.

16. Tidermark J, Ponzer S, Tengstrand B, Cederholm T. Liquid supplementation and nandrolon to elderly women after a femoral neck fracture (Abstract). Clin Nutr 2002; 21S: S58.

17. Yeh SS, Wu SY, Lee TP, Olson JS, Stevens MR, Dixon $\mathrm{T}$, et al. Improvement in quality-of-life measures and stimulation of weight gain after treatment with megestrol acetate oral suspension in geriatric cachexia: results of a double blind, placebo-controlled study. J Am Geriatr Soc 2000; 48: 485-92.

Tommy Cederholm, , MD, PhD

B56, Department of Geriatric Medicine, Huddinge University Hospital, SE-14186 Stockholm, Sweden

E-mail: tommy.cederholm@neurotec.ki.se 